\title{
A Case of Rarest Presentation of CNS Tuberculosis as Disseminated Miliary Tuberculoma Brain
}

Rahul Soni ( $\nabla$ drrahulsoni1977@gmail.com )

Base Hospital Delhi Cantt https://orcid.org/0000-0002-0150-1398

\section{Manish Saha}

Base Hospital Delhi cantt

Aman miglani

Base Hospital Delhi Cantt

Jayita Debnath

Clinical Biochemistry, Delhi

Case report

Keywords: Tuberculoma, Tuberculosis, Acid Fast bacilli, MRI

Posted Date: May 27th, 2021

DOI: https://doi.org/10.21203/rs.3.rs-523117/v1

License: (c) (i) This work is licensed under a Creative Commons Attribution 4.0 International License. Read Full License 


\section{Abstract}

Introduction: Disseminated miliary forms of tuberculoma in brain are rarest form of CNS tuberculosis. It is one of the differential diagnoses of ring enhancing multiple brain lesions. We are reporting an interesting case of 28 year old lady with disseminated tuberculosis with miliary brain tuberculomas.

Case presentation: 28 yrs lady with no known previous co-morbidities presented with low grade evening rise in temperature and mild to moderate continues global headache for 10 days associted with nausea and recurrent vomiting, and weight loss of $4 \mathrm{~kg}$. Her ESR, blood count, liver and renal function tests were within normal values. HIV was negative by ELISA. Sputum for Acid Fast bacilli was +3 positive for mycobacterium tuberculosis bacilli. Cerebrospinal Fluid cytological analysis was normal and ADA was 9.1 U/L. Urine microscopic examination was positive for +++ MTB. Chest $X$ ray showed bilateral military opacities. High Resolution CT of Chest was suggestive of miliary distribution of nodules in bilateral lungs. MRI Brain showed discrete ring enhancing lesions scattered in left temporal lobe, and right frontal lobe are located at grey-white matter junctions. In infra- tentorial lesion were at the level of pons, mid brain, inferior cerebellar peduncle and bilateral cerebellar hemispheres. Few of the lesions in right precentral gyrus showing associated mild perilesional edema.

Conclusion: Clinician should consider miliary tuberculosis brain in tuberculosis endemic region, in a clinical setting where atypical multiple ring enhancing lesions even with non inflammatory CSF but with presence of other markers of tuberculosis is present.

\section{Introduction}

Central nervous system (CNS) tuberculoma are one of the first differential diagnosis of enhancing intraaxial lesion in most of developing countries. Disseminated miliary forms of tuberculoma in brain are rarest form of CNS tuberculosis. It is one of the differential diagnoses of ring enhancing multiple brain lesions. We are reporting an interesting case of 28 year old lady with disseminated tuberculosis with miliary brain tuberculomas, miliary pulmonary involvement and urinary tract involvement.

\section{Case Presentation}

28 yrs lady with no known previous co-morbidities presented with low grade evening rise in temperature and mild to moderate continues global headache for 10 days. Headache was insidious in onset and gradually progressive, and associted with nausea and recurrent vomiting. She had weight loss for $4 \mathrm{~kg}$ over a period of 1 month due to loss of appetite. She denies history of tuberculosis in any other family members. On physical examination there was no pallor or lymphadenopathy or clubbing or any cutaneous markers of tuberculosis. No neck rigidity and fundoscopy was normal. Chest and abdomen examination was unremarkable. Investigation showed raised ESR of $18 \mathrm{~mm}$ fall after 1 hour. Routine blood count, liver and renal function tests were within normal values. HIV was negative by ELISA. Sputum for Acid Fast bacilli was +3 positive for mycobacterium tuberculosis bacilli (MTB). Cerebrospinal Fluid 
(CSF) analysis showed 02 WBCs (both lymphocytes), protein was $40 \mathrm{mg} / \mathrm{dL}$ and ADA was $9.1 \mathrm{U} / \mathrm{L}$. Urine microscopic examination was also positive for +++ MTB. Chest $X$ ray showed bilateral military opacities (Figure1). High Resolution CT of Chest was suggestive of miliary distribution of nodules in bilateral lungs (Figure2). Non contrast CT head was suggestive of ill defined right frontal hypodensity without mass effect with a small focal hyperdensity within suggestive of a granuloma. MRI Brain showed discrete ring enhancing lesions scattered in left temporal lobe, and right frontal lobe are located at grey-white matter junctions. In infra- tentorial lesion were at the level of pons, mid brain, inferior cerebellar peduncle and bilateral cerebellar hemispheres. Few of the lesions in right precentral gyrus showing associated mild perilesional edema (Figure 3 to 9). She was managed as case of disseminated tuberculosis with antitubercular treatment. She responded to treatment and within one week fever subsided and started gaining weight after 4 weeks of treatment.

\section{Discussion}

Tuberculosis is a challenging disease even in developed nations with even with advance medical facilities. One of most potentially lethal form is miliary form, which can be either pulmonary or brain. It needs early diagnosed and treatment. Earlier miliary pulmonary TB used to occur mainly in children however lately it's occurrence has significantly increased in younger and elderly population. ${ }^{(1)}$ Endogenous reactivation of TB and massive lymphogenous and hematogenous dissemination is seen in people who are on immunosuppressive, immunomodulating, and cytotoxic drug therapy. The microbiological confirmation of any other form of TB is unsatisfactory in comparison to pulmonary TB.

Even in miliary tuberculosis microbiological confirmation on sputum, sensitivity reduced to one third. ${ }^{(2)}$ In our patient sputum and urine both were positive for MTB bacilli. However, her CSF did not show Tubercular bacilli.

Any ring enhancing lesion in brain is a diagnostic challenge for clinicians because most of these patients come with similar clinical syndromes. Contrast MRI brain is one of best investigation in diagnosis of ring enhancing lesion brain. MRI sensitivity is also very high for diagnosing other form of tuberculosis like tubercular exudative leptomeningitis. ${ }^{(3)}$ However, $n$ then even some time it is very difficult in differentiating from other causes ring enhancing lesion like Neurocysticercosis, fungal granuloma, Toxoplasma and even metastatic malignancies on plan MRI Brain when the lesion are very small in size. Closest differential diagnosis of miliary brain tuberculoma is multiple Neurocysticercosis.

Tuberculomas are commonly more than $20 \mathrm{~mm}$ in size however in miliary tuberculoma are less than 20 $\mathrm{mm}$ in size so difficult to differentiate from Neurocysticercosis on the bases of size. ${ }^{(4)}$ On non contrast CT scan these lesions can easily be missed because of small size. Another important character is that, these lesions have very less perilesional edema as compare to classical tuberculoma which has disproportionate perilesional edma. In tuberculoma restriction diffusion is seen on diffusion weighted MRI images. ${ }^{(5)}$ However, in miliary tuberculoma diffusion restriction are not seen. Tuberculoma are generally conglomerate lesion, but miliary tuberculomas are discrete spreads out lesion. With all these 
features it is very difficult to differentiate from multiple Neurocysticercosis. In our case, patient had multiple early mild disc enhancing lesions at the level of left temporal lobe, midbrain and right frontal lobe, pons, inferior cerebellar peduncle and bilateral cerebellar hemispheres. There was no significant perilesional edema on T2 and Flair images. Even there was no conglomerate lesion seen.

Magnetic resonance spectroscopy (MRS) is a specific MRI sequence which can help distinguish tubercular lesion from Neurocysticercosis. Elevated lactate (LA) peak, diminish N Acetyl Aspartate (NAA) and creatinine peak, and a choline/creatinine ratio of more than one is seen in tuberculoma. ${ }^{(6)}$ Whereas in NCC these is elevated lactate and proteins such as glutamate, alanine, succinate, glycine levels with some reduction of NAA and creatinine is seen. ${ }^{(7)}$ We have not done MRS sequence since in our patient's urine and sputum has shown mycobacterium tuberculosis.

Serological markers in CSF are also helpful in case of making diagnosis. Even though CSF of our patient was normal, but ADA was significantly raised. High ADA is also suggestive of CNS tuberculosis. CSF can be normal in tuberculoma brain since there is no inflammatory response at meanings.

\section{Conclusion}

Clinician should consider miliary tuberculosis brain in TB endemic region, in a clinical setting where atypical multiple ring enhancing lesions even with non-inflammatory CSF but with presence of other markers of tuberculosis is present.

\section{Abbreviation}

Mycobacterium Tuberculosis Bacilli (MTB) Adenosine Deaminase Level (ADA), Magnetic resonance spectroscopy (MRS).

\section{Declarations}

Ethics approval and consent to participate: Ethical Approval from Ethical committee named "Institutional Ethical committee Base Hospital, Delhi Cantt" and written informed consent was obtained from patient.

Consent for publication: Consent was obtained for publication.

Availability of data and material: From medical records of hospital.

Competing interests: The authors declare that they have no competing interests.

Funding: It is certified that author's team are not currently in receipt of any research funding.

Authors' contributions: All authors have read and approved the manuscript, and ensure that this is the case. RS was treating when patient was in ICU and did the research necessary for its production, MS was 
radiologist involved in this case and wrote the article, AM was resident medicine and elaboration of the case description and JD in the article review.

Acknowledgements: Not applicable

\section{References}

1. Sharma SK, Mohan A, Sharma A, Mitra DK. Miliary tuberculosis: new insights into an old disease. Lancet Infect Dis 2005;5:415-30.

2. Sharma SK, Mohan A, Sharma A. Challenges in the diagnosis \& treatment of miliary tuberculosis. Indian J Med Res 2012;135:703-30.

3. Rock RB, Olin M, Baker CA, Molitor TW, Peterson PK. Central nervous system tuberculosis: pathogenesis and clinical aspects. Clin Microbiol Rev 2008;21:243-61, table of contents.

4. Bharat-Bhushan Sharma, Shashi Sharma. Neurocysticercosis (NCC) vs Central Nervous System (CNS) Tuberculoma in Children - Dilemma over Clinico - Radiological Diagnosis?. Open Journal of Pediatrics, 2016, 6, 245-251. DOI: 10.4236/ojped.2016.63034.

5. Gupta RK PM, Mishra AM, Husain M, Prasad KN, Husain N. Role of diffusion weighted imaging in differentiation of intracranial tuberculoma and tuberculous abscess from cysticercus granulomas-a report of more than 100 lesions. Eur J Radiol. 2005. 55(3):384-92.

6. Seth R, Kalra V, Sharma U, Jagannathan N. Magnetic resonance spectroscopy in ring enhancing lesions. Indian Pediatr. 2010 Sep;47(9):803-4.

7. S Chawla, S Kumar, M Garg, R Kumar, R Roy, R K Gupta. Metabolite pattern of Cysticercus cellulosae metacestode from different predilection sites of swine using proton magnetic resonance spectroscopy. Parasite. 2004 Jun;11(2):161-7. doi: 10.1051/parasite/2004112161.

\section{Figures}




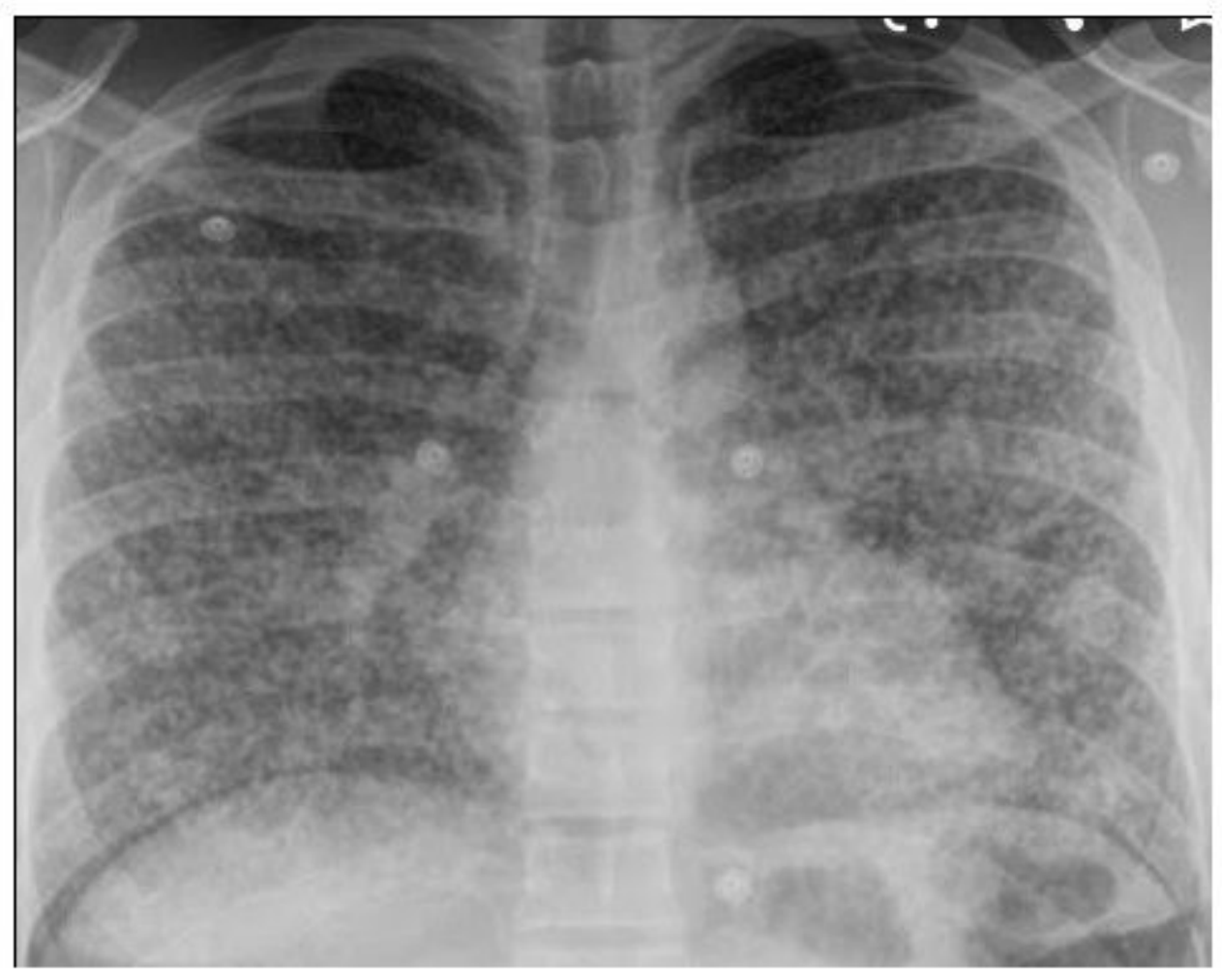

Figure 1

Chest X Ray PA view: Miliary shadows noted in bilateral lung fields.

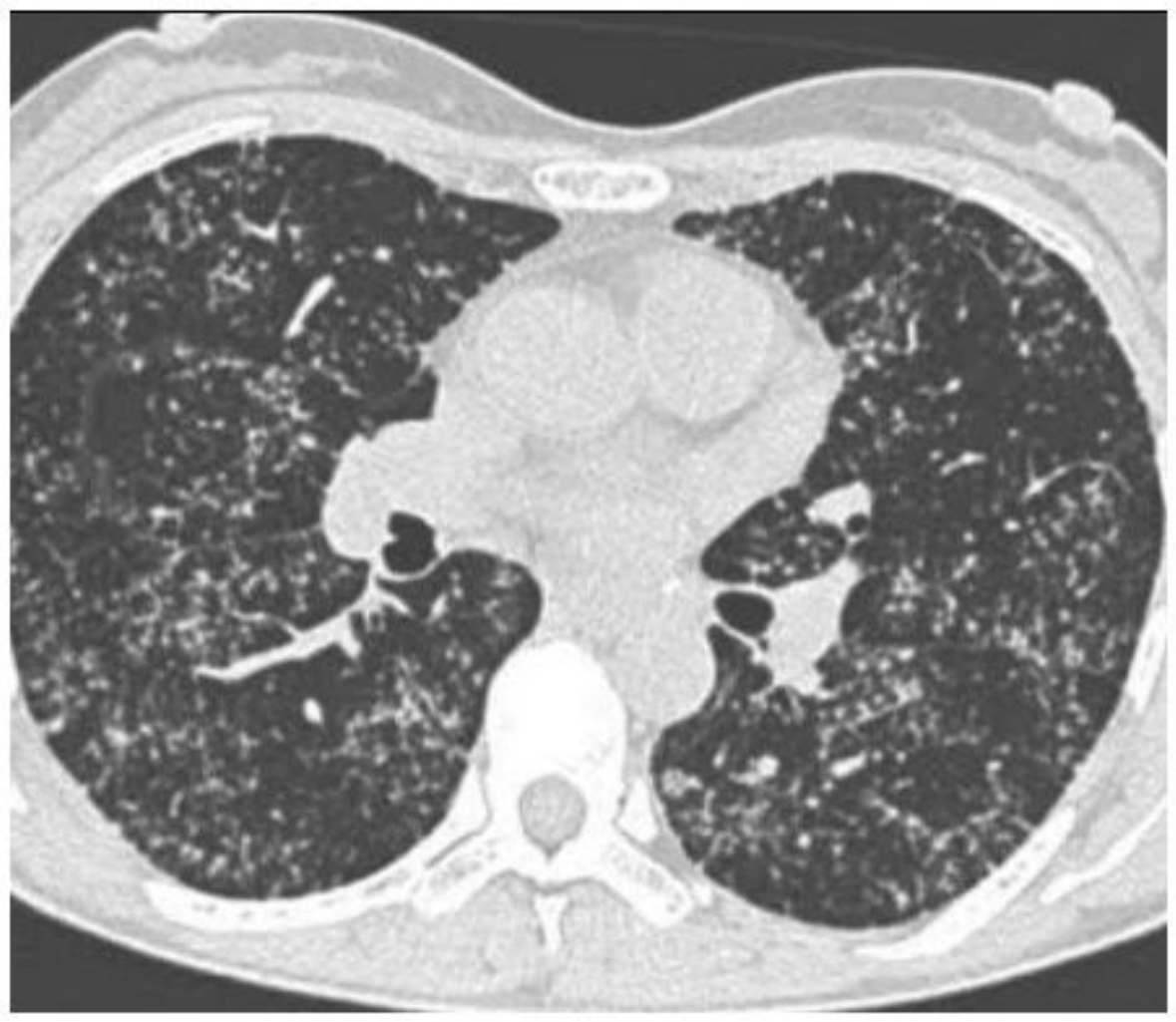

Figure 2 
HRCT chest at the level of bronchus intermedius showing miliary distribution of nodules in bilateral lungs.

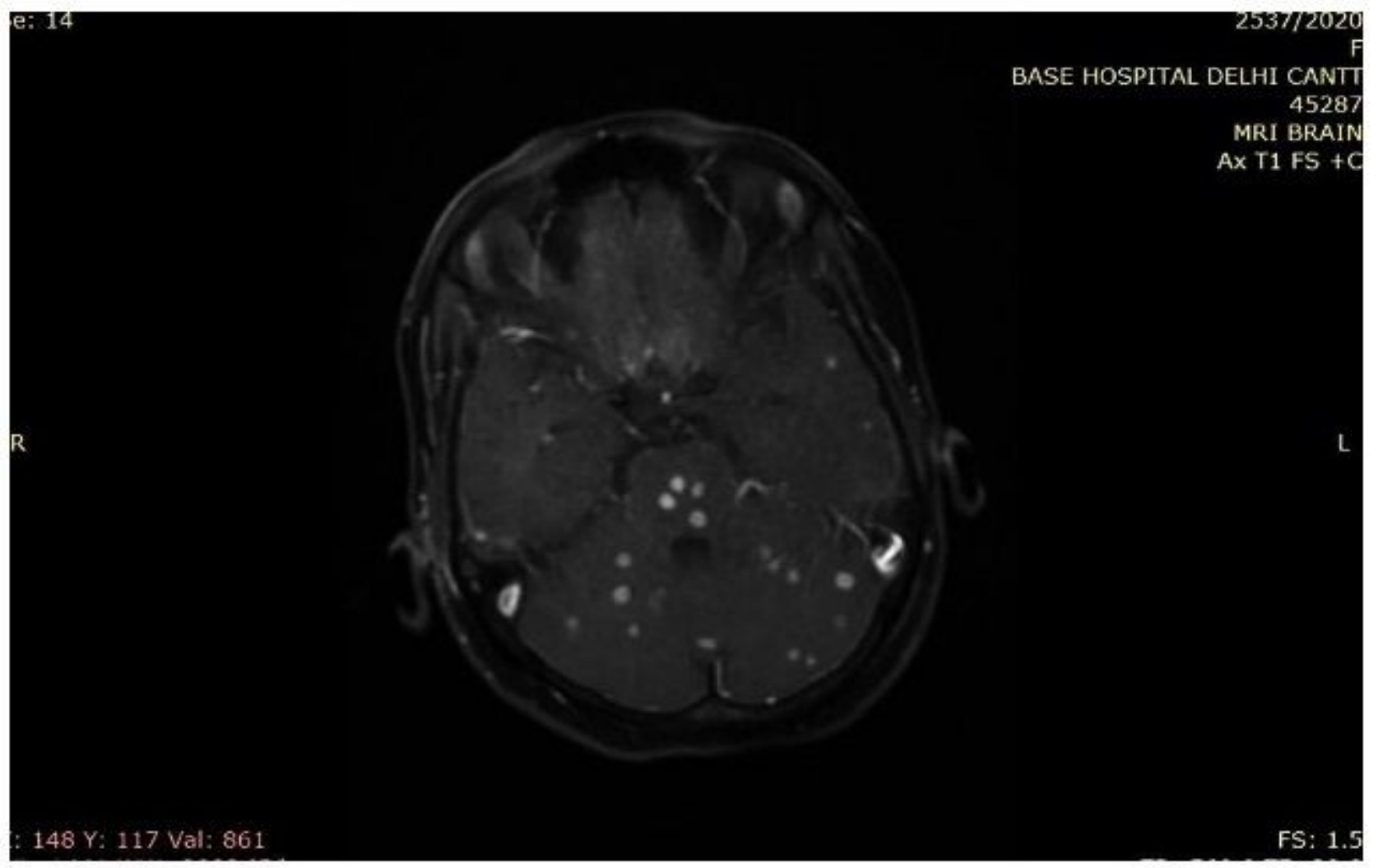

\section{Figure 3}

MRI T1 Contrast images showing multiple well defined round ring and solid enhancing lesions are noted at the level of pons, inferior cerebellar peduncle and bilateral cerebellar hemispheres. 


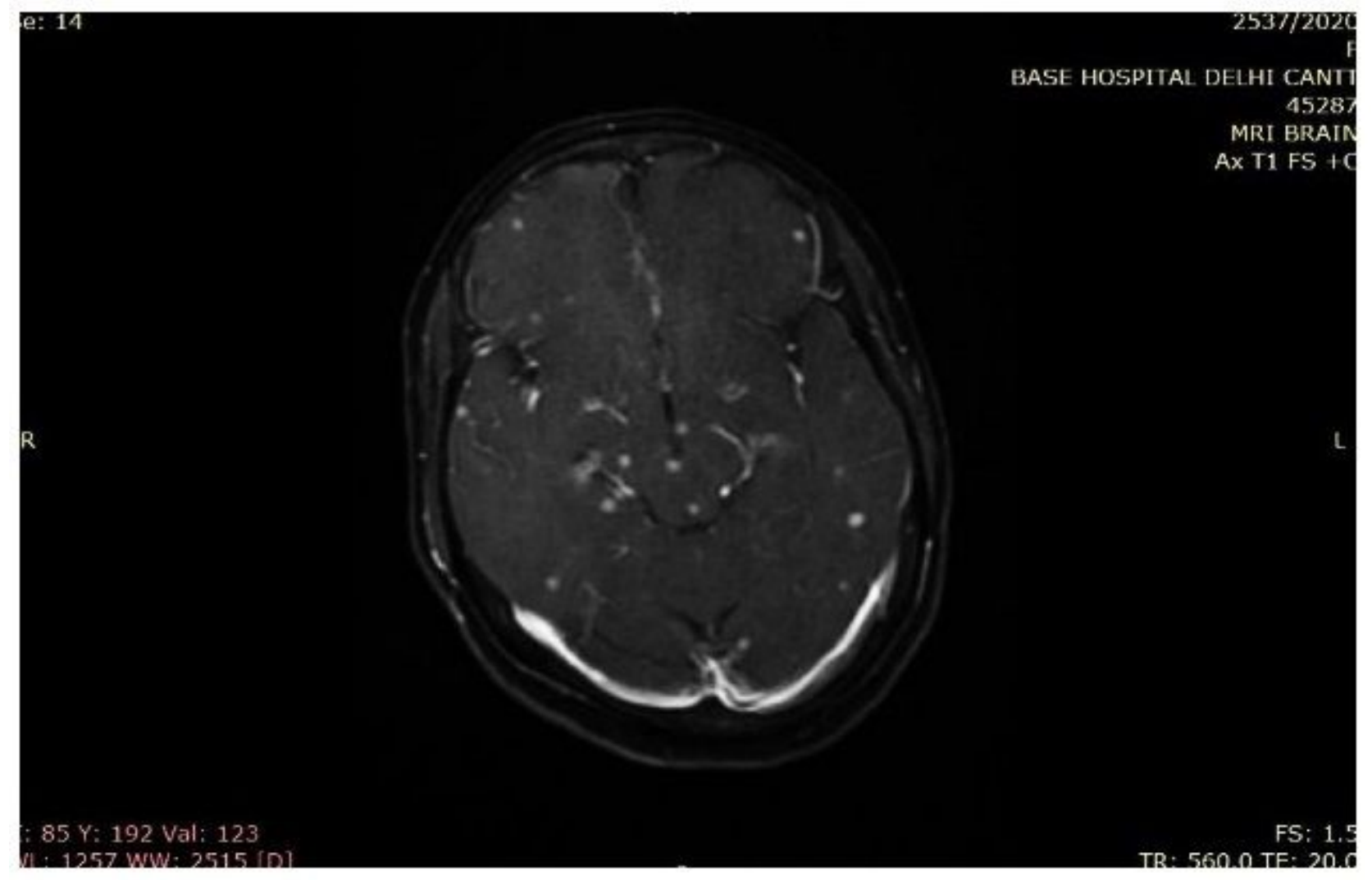

\section{Figure 4}

MRI T1 contrast images showing multiple 1.5 $-2 \mathrm{~mm}$ round solid enhancing lesions are noted in left temporal lobe, midbrain and right frontal lobe. The lesions noted in cerebral hemispheres are located at grey-white matter junctions. 


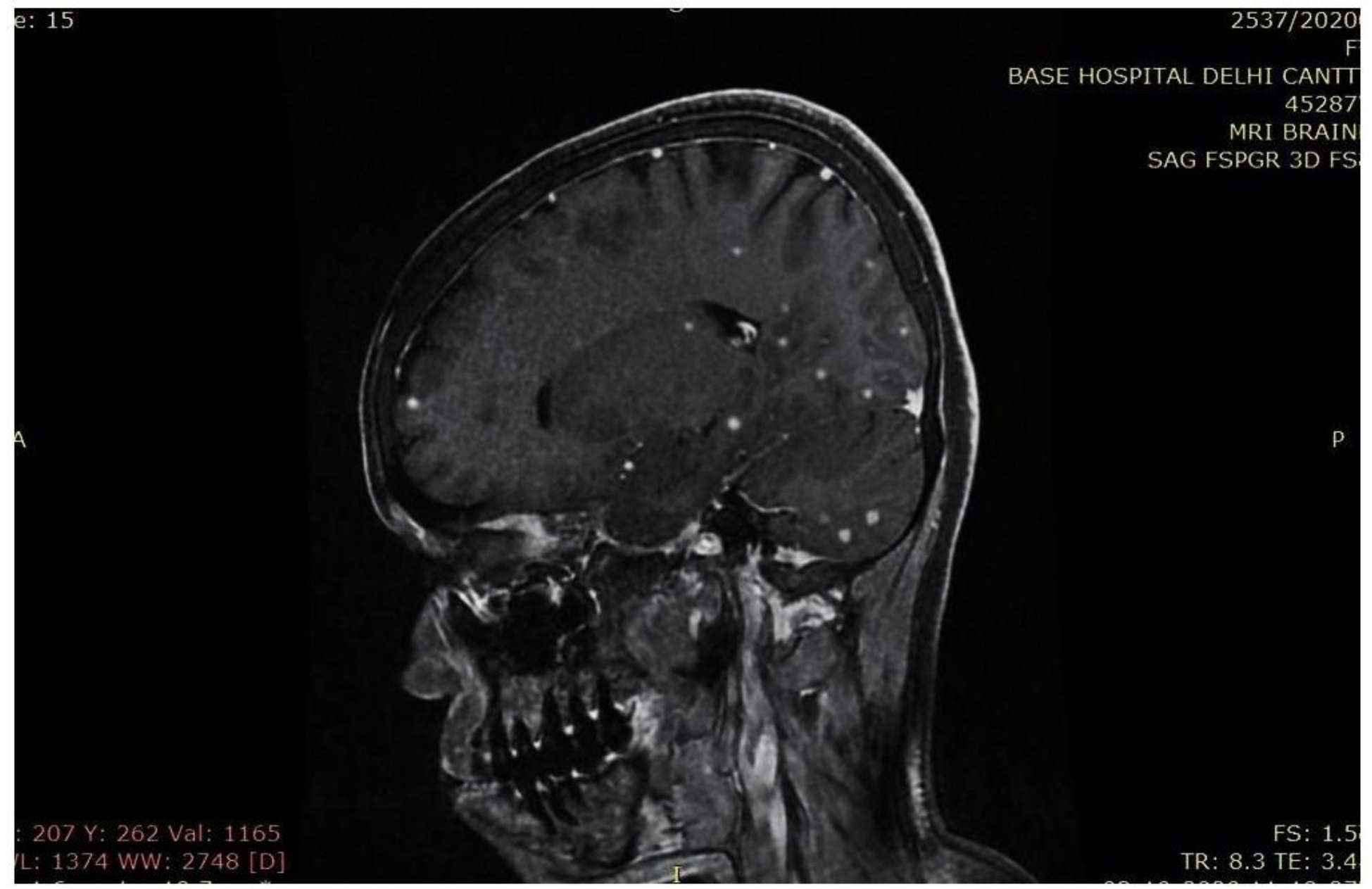

\section{Figure 5}

Multiple solid enhancing lesions are noted in supratentorial and infratentorial compartments as well as along duramater. 


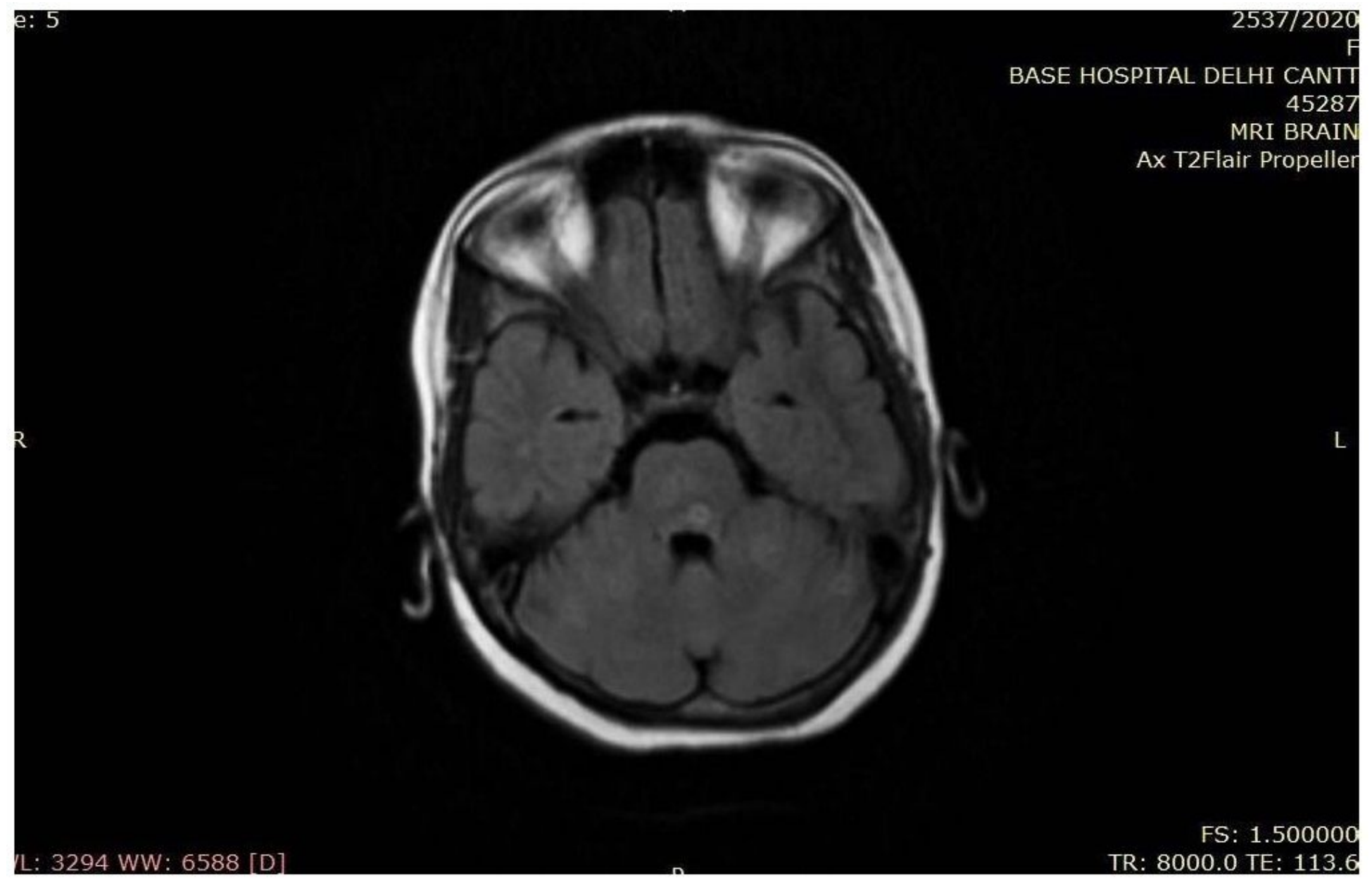

\section{Figure 6}

MRI Axial FLAIR image at the level of inferior cerebellar peduncle - showing subtle heterogeneous intensity round ring shaped lesions in cerebellar hemispheres and brachium pontis. The lesions show a FLAIR hyperintense margin with central hypointensity. 


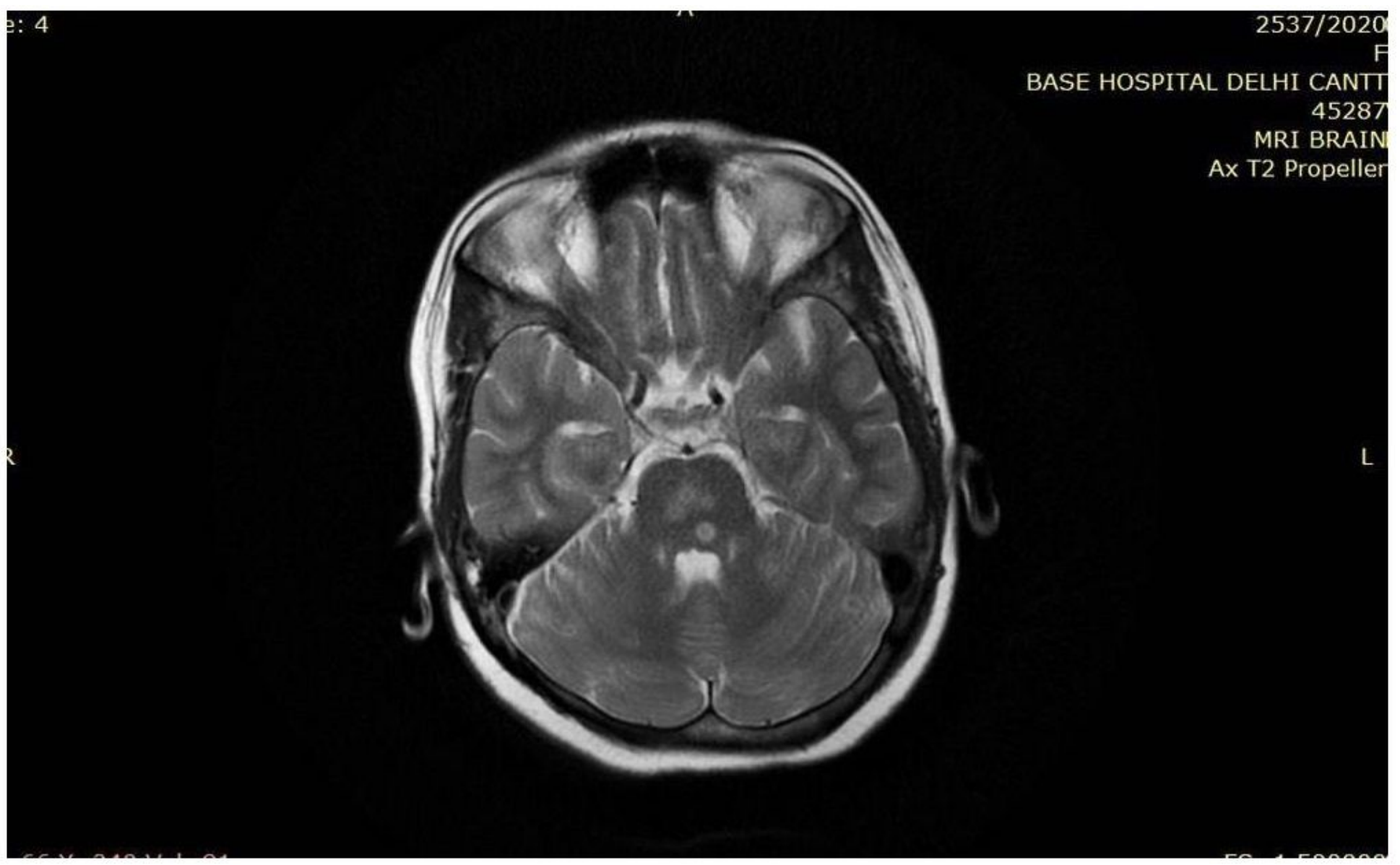

\section{Figure 7}

Axial T2W image at mid-pontine level - showing discrete heterogeneous signal intensity ring shaped lesions. The lesions show hyperintense margin with hypointense centre. The dorsally located lesion showing a well defined T2 WI hyperintense margin while the ventrally located lesions showing haziness suggestive of mild perilesional edema. 


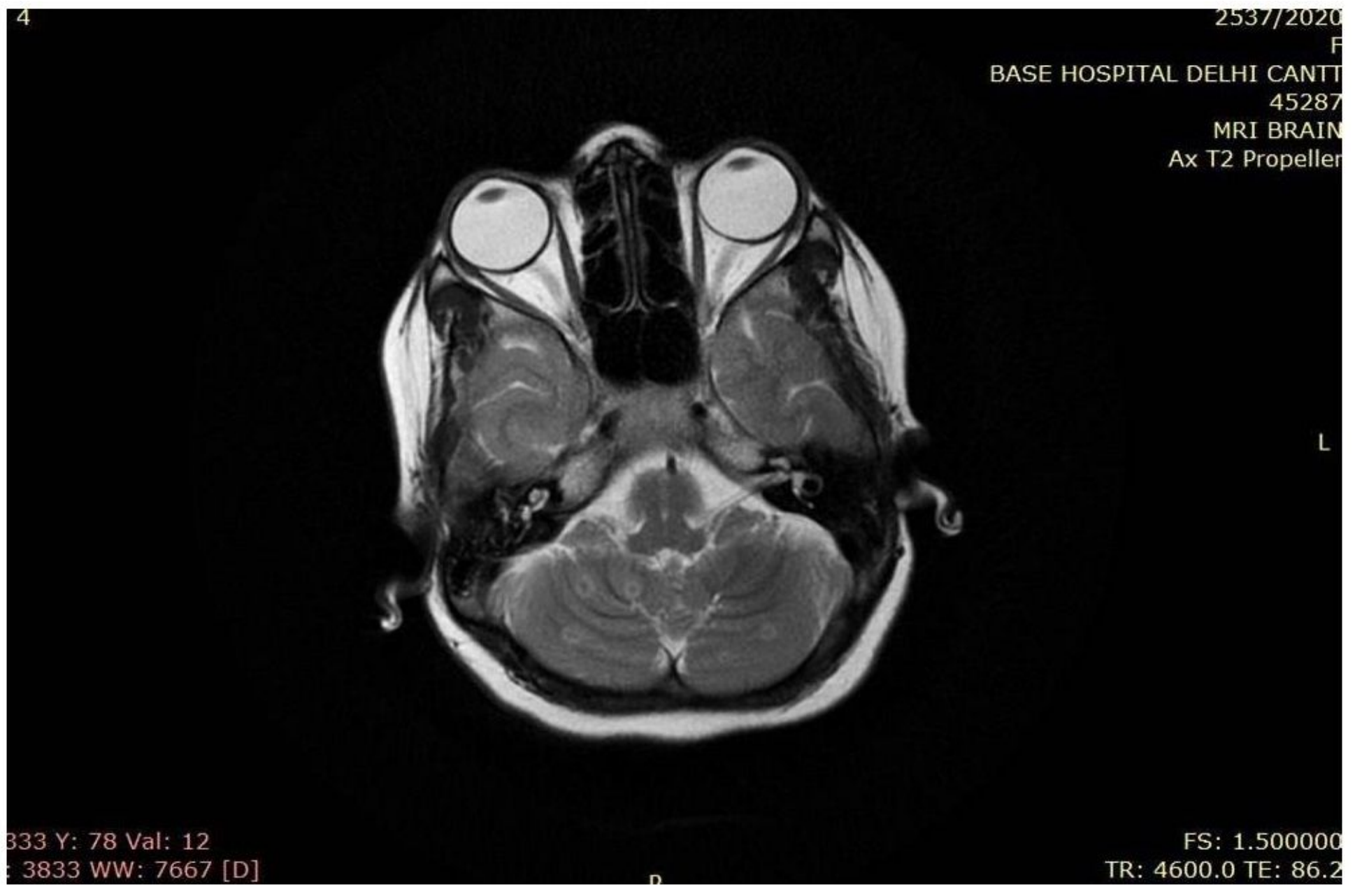

\section{Figure 8}

Axial T2WI image at the level of ponto-medullary junction - showing heterogeneous signal intensity lesions with hyperintense margin and hypointense centre. 


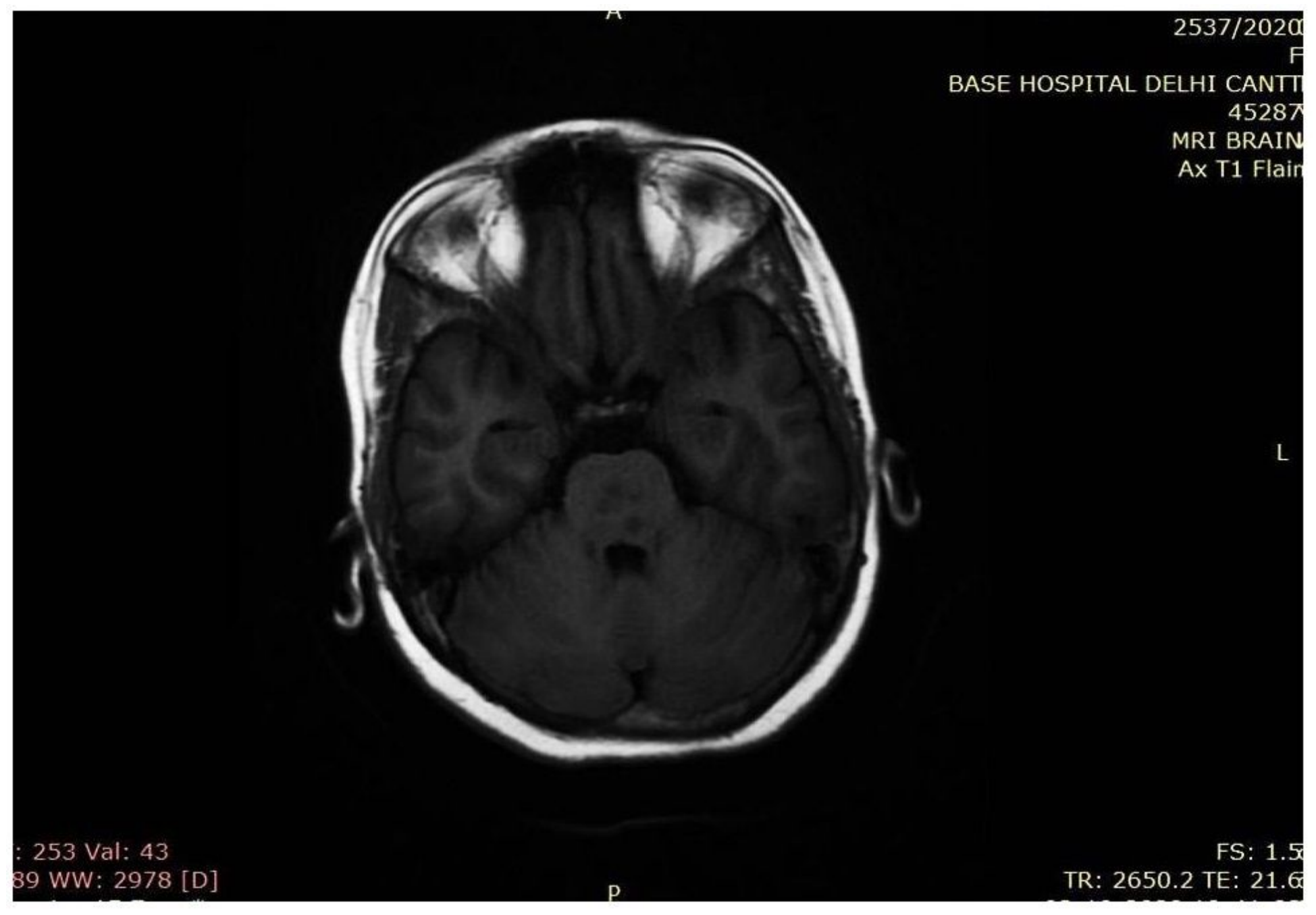

\section{Figure 9}

Axial T1 image at mid-pontine level; showing discrete round homogeneously hypointense signal intensity lesions. 